\title{
Estructura poblacional de Chiton articulatus en las islas Pájaros y Venados de la bahía de Mazatlán, Sinaloa, México
}

\author{
Population structure of Chiton articulatus from Pájaros and Venados islands of Mazatlán Bay, \\ Sinaloa, Mexico
}

Luis Miguel Flores-Campaña ${ }^{1 *}$, Marco Antonio González-Montoya ${ }^{1}$, Mónica Anabel Ortiz-Arellano ${ }^{1,2}$ y Juan Francisco Arzola-González ${ }^{1}$

${ }^{1}$ Facultad de Ciencias del Mar, Universidad Autónoma de Sinaloa, A.P. 610, Mazatlán, Sinaloa, México.

${ }^{2}$ Centro Interdisciplinario de Investigación para el Desarrollo Integral Regional. Instituto Politécnico Nacional, Guasave, Sinaloa. México.

*Correspondencia: lcampana@uas.uasnet.mx

\begin{abstract}
Resumen. El propósito del presente trabajo fue contribuir al conocimiento biológico y pesquero de Chiton articulatus, especie que se captura artesanalmente en las costas de Mazatlán, Sinaloa. Se realizaron recolectas bimestrales en la zona intermareal de las islas Pájaros y Venados, de febrero a diciembre de 2000, durante las mareas más bajas. Las recolectas se realizaron en 4 sitios distintos, 2 en la zona protegida frente a la bahía Mazatlán y 2 en la zona expuesta de ambas islas; los ejemplares se trasladaron al laboratorio para su identificación y registro de dimensiones corporales con un vernier $( \pm 0.1 \mathrm{~mm})$ y una balanza $( \pm 0.001 \mathrm{~g})$. Se analizaron 1236 organismos, su longitud total $(\mathrm{Lt})$ osciló entre 11.2 y $86.8 \mathrm{~mm}$, su altura (Al) fue de 2.0 a $48.1 \mathrm{~mm}$, su anchura (An) varió de $7.1 \mathrm{~mm}$ a $48.1 \mathrm{~mm}$ y su peso total (Pt) entre $0.1 \mathrm{y} 53.8 \mathrm{~g}$. La mayor parte de la población se distribuyó entre los 31 y $64 \mathrm{~mm}$, con una moda de $41.1 \mathrm{~mm}$ y media de $45.6 \pm 8.5 \mathrm{~mm}$ de Lt. En la zona expuesta de isla Pájaros se presentaron mayores tallas, mientras que las menores se encontraron en la zona protegida de isla Venados; esta diferencia puede explicarse tanto por el grado de exposición al oleaje como por la presión de pesca, que se relaciona con la dificultad de acceso en cada zona. Las relaciones biométricas entre las tallas fueron de tipo lineal $\{\mathrm{An}=0.5423(\mathrm{Lt})+2.7594, \mathrm{Al}=0.2316(\mathrm{Lt})-0.4576 \mathrm{y} \mathrm{Al}=0.3807(\mathrm{An})-0.3832\}$ y entre talla y peso fueron de tipo potencial $\left\{\mathrm{Pt}=0.0001(\mathrm{Lt})^{2.8404}, \mathrm{Pt}=0.0005(\mathrm{An})^{2.8807}\right.$ y $\left.\mathrm{Pt}=0.0329(\mathrm{Al})^{2.3236}\right\}$; los valores de la pendiente (b) de las relaciones entre la talla y el peso fueron cercanos a 3, por lo cual se considera que su crecimiento es de tipo isométrico. La relación entre el $\mathrm{Pt}$ y el peso del pie $(\mathrm{Pp})$ fue $\{\mathrm{Pt}=4.8778(\mathrm{Pp})+1.0933\}$. La proporción que se aprovecha de cada individuo es casi del $17 \%$ de su Pt. La captura de C. articulatus es una actividad de subsistencia que diversos grupos realizan de manera temporal o complementaria de otras actividades. No existe ningún tipo de normatividad y/o vigilancia sobre su captura y comercialización.
\end{abstract}

Palabras clave: moluscos, Chiton articulatus, Pacífico este tropical, islas, distribución de tallas, pesca.

\begin{abstract}
This work represents a contribution to the knowledge of the basic and fishery biology of Chiton articulatus, which is exploited by amateur fishermen along the coast of Mazatlán, Sinaloa. Collections were made every two months from February to December of 2000 at four different sites in the intertidal zone of Pájaros and Venados islands, during the lowest tide. Two sites were in the protected zone facing Mazatlán Bay and two in the exposed zones of both islands. All specimens were identified and measured in the laboratory where body size and weight were obtained with a caliper $( \pm 0.1 \mathrm{~mm})$ and scale $( \pm 0.001 \mathrm{~g})$ respectively. The total length $(\mathrm{Lt})$ of the 1,236 specimens examined varied between 11.2 and $86.8 \mathrm{~mm}$, height (Al) between 2.0 and $48.1 \mathrm{~mm}$, width (An) from $7.1 \mathrm{~mm}$ to $48.1 \mathrm{~mm}$ and total weight (Pt) from $0.1 \mathrm{~g}$ to $53.8 \mathrm{~g}$. Size distributions showed that most of the population was between Lt of $31-64 \mathrm{~mm}$ with a modal Lt of $41.1 \mathrm{~mm}$ and mean of $45.6 \pm 8.5 \mathrm{~mm}$. The larger sizes were recorded on the exposed zone of Pajaros island and the smaller sizes were found in the protected zone of Venados island. This difference can be explained by wave exposure and differences in fishery pressure related to the ease of access to each zone. Biometric relationships between sizes were linear $\{\mathrm{An}=0.5423(\mathrm{Lt})+2.7594, \mathrm{Al}=0.2316(\mathrm{Lt})-0.4576$ and $\mathrm{Al}=0.3807(\mathrm{An})-0.3832\}$ and between size and weight were exponential $\left\{\mathrm{Pt}=0.0001(\mathrm{Lt})^{2.8404}, \mathrm{Pt}=0.0005(\mathrm{An})^{2.8807}\right.$ and $\left.\mathrm{Pt}=0.0329(\mathrm{Al})^{2.3236}\right\}$; the values of slope $(\mathrm{b})$ between size and
\end{abstract}


weight relationships were close to 3, indicating isometric growth. The relationship between $\mathrm{Pt}$ and foot weight (Pp) was $\{\mathrm{Pt}=4.8778(\mathrm{Pp})+1.0933\}$, which means that the portion of each specimen used was $17 \%$ of Pt. Capture of C. articulatus is a subsistence activity for different groups for temporary relief in times of poor catches or to complement earnings from other activities. There are no regulations governing the harvesting and commercialization of this mollusk.

Key words: molluscs, Chiton articulatus, Eastern Tropical Pacific, islands, size distribution, fishery.

\section{Introducción}

Las islas Pájaros, Venados y Lobos de la bahía de Mazatlán, México, son importantes sitios de captura de varias especies de crustáceos, peces y moluscos. Entre estos últimos se encuentra Chiton articulatus Sowerby, 1832, un molusco poliplacóforo, conocido en la localidad como cucaracha de mar. No obstante que la captura de esta especie es una actividad de subsistencia que diversos grupos realizan de manera temporal o complementaria de otras actividades, es poca la información que se tiene sobre su biología.

El conocimiento científico de los moluscos de México ha sido abordado por algunos investigadores, aunque siempre se ha dado mayor énfasis a los estudios sobre su taxonomía y distribución geográfica, así como a la dinámica de poblaciones de especies de interés comercial con la finalidad de mejorar las condiciones de su explotación (Reguero y García-Cubas, 1989; González, 1993; Hendrickx y Toledano-Granados, 1994; Fischer et al., 1995). Sin embargo, han sido poco estudiados otros aspectos que son fundamentales cuando se considera la necesidad de disponer de información acerca de las especies que se distribuyen en las costas mexicanas, tanto de las que son objeto de explotación, como de las que forman parte de las comunidades silvestres con importancia ecológica.

Los estudios sobre los quitones de las costas mexicanas se refieren principalmente a listados taxonómicos con información sobre su morfología y distribución geográfica (Keen, 1971; Clark, 2000; Reyes-Gómez y SalcedoVargas, 2002; Reyes-Gómez, 2004). Los trabajos que exclusivamente se refieren a quitones son escasos y versan sobre distribución, abundancia y relaciones biométricas de C. articulatus (Rojas-Herrera, 1988; Holguin-Quiñones y Michel-Morfin, 2002) y la descripción de la riqueza de especies de la clase Poliplacophora en una laguna arrecifal de Quintana Roo (Cervera-Rodríguez et al., 2004).

Las investigaciones realizadas en el ámbito internacional son más numerosas y se han enfocado sobre las diversas especies de quitones, en aspectos relacionados con su taxonomía (Montoya, 1983; O`Neill, 1985; Kaas y Van Belle, 1998; Slieker, 2000), morfología (Baxter y Jones 1981; Cameron, 1982), biología reproductiva (Nagabhushanam y Deshpande, 1982; Deshpande y
Nagabhushanam, 1983; Sakker, 1984) y dinámica poblacional y ecología (Cruz y Sotela, 1984; Otway, 1994; Grayson y Chapman, 2004; Kelaher y Cole, 2005).

El propósito del presente trabajo es contribuir al conocimiento biológico y pesquero de C. articulatus y aportar información sobre su estructura poblacional y tallas alcanzadas en diferentes zonas de las islas Venados y Pájaros, así como describir aspectos generales sobre su captura y comercialización en la región de Mazatlán, Sinaloa.

\section{Material y métodos}

Las 3 islas se localizan entre $\operatorname{los} 23^{\circ} 13^{\prime} 20^{\prime \prime}$ y $23^{\circ} 15^{\prime}$ 17 " N y los $106^{\circ} 27^{\prime} 59^{\prime \prime}$ y $106^{\circ} 28^{\prime} 41^{\prime \prime}$ O, frente a la bahía de Mazatlán, al sureste del Golfo de California. Tienen una orientación lineal norte-sur y reciben los nombres de Pájaros, Venados y Lobos, respectivamente. Las dos últimas están unidas por una franja angosta de arena que queda descubierta en marea baja (Fig. 1).

Las recolectas de C. articulatus se llevaron a cabo en la zona intermareal rocosa de las islas Pájaros y Venados, de febrero a diciembre del 2000, con un intervalo de muestreo bimestral durante las mareas más bajas. Se establecieron 4 estaciones de recolecta en zonas diferentes de estas islas; una en la zona protegida de cada isla, frente a la bahía de Mazatlán con un oleaje de baja energía y otra en la zona expuesta de cada isla, frente al mar abierto, caracterizada por un fuerte oleaje y una topografía muy abrupta.

Los organismos se recolectaron en forma manual utilizando espátulas o cuchillos, ya que se encontraban adheridos a las rocas en la zona de rompientes. Los ejemplares recolectados se depositaron en bolsas de plástico previamente etiquetadas para luego trasladarlos al Laboratorio de Invertebrados y Ecología del Bentos (LIEB) de la Facultad de Ciencias del Mar, Universidad Autónoma de Sinaloa, donde se les tomaron medidas en vivo, ya que estos organismos tienden a contraerse, lo que dificulta su manipulación y el registro de sus dimensiones corporales.

La identificación de C. articulatus se hizo utilizando las características descritas en Keen (1971) y Brusca (1980). En el laboratorio, los quitones se depositaron en charolas 


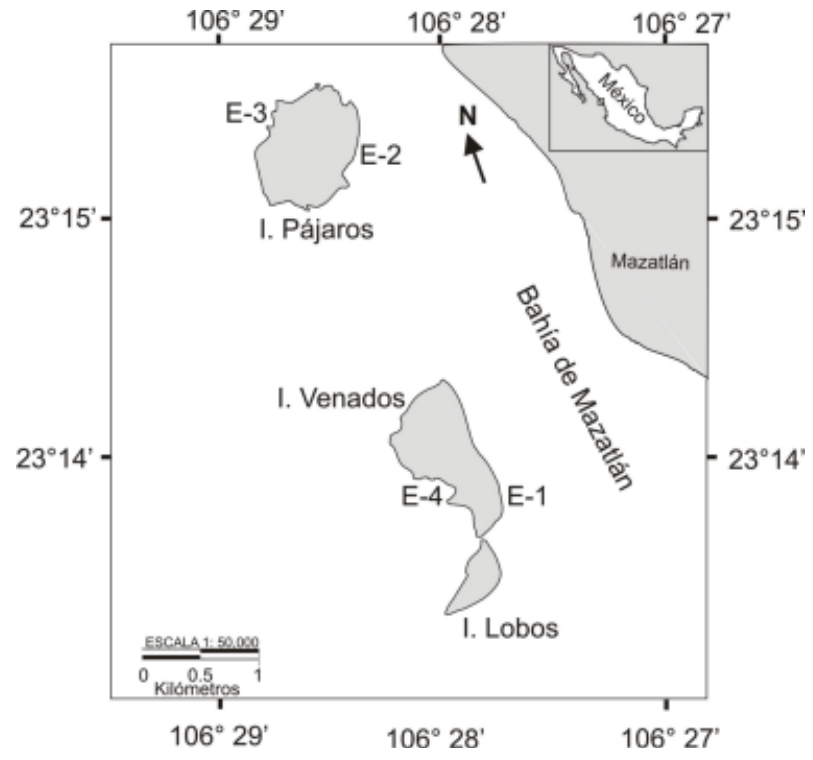

Figura 1. Estaciones de recolecta en las islas de la bahía de Mazatlán, Sinaloa, México.

previamente etiquetadas para registrar las biometrías correspondientes; longitud total (Lt), altura (Al) y anchura (An), utilizando un vernier digital marca Mitutoyo $( \pm 0.1$ $\mathrm{mm})$. El peso total $(\mathrm{Pt})$ y el peso del pie $(\mathrm{Pp})$ se registraron con una balanza digital marca Ohaus $( \pm 0.001 \mathrm{~g})$.

La Lt se tomó desde el inicio de la parte anterior del cinturón en la placa cefálica hasta la parte final del mismo en la placa anal. La An se midió en forma transversal, de lado a lado del cinturón, entre la cuarta y quinta placa; por último, la $\mathrm{Al}$ se registró en la parte media del animal, desde la base del pie hasta la región dorsal de la cuarta y quinta placa.

Los datos de Lt se utilizaron para elaborar histogramas de frecuencia para cada sitio de recolecta y para cada isla. Los histogramas se construyeron con un intervalo de talla de $2 \mathrm{~mm}$ de Lt. Se aplicaron las pruebas estadísticas de normalidad de Lilliefors y homoscedasticidad de Bartlett. Posteriormente, se realizó una prueba no paramétrica, a través del análisis de varianza de una vía de Kruskal-Wallis, la prueba de comparaciones múltiples no paramétrica de Dunn y la prueba $U$ de Mann-Whitney para percibir diferencias entre las longitudes (Zar, 1996), en todos los casos se utilizó el programa Statistica for Windows 2000.

Se utilizó la información registrada sobre longitud, altura, anchura y peso, para obtener las relaciones entre las tallas (longitud, altura y anchura) y entre las tallas y el peso. Para ello se construyeron los diagramas de dispersión correspondientes y se ajustaron a modelos matemáticos de acuerdo con la tendencia observada, según Ricker (1975).
Los modelos de las relaciones biométricas obtenidas se ajustaron utilizando el método de mínimos cuadrados y se realizaron las pruebas estadísticas del coeficiente de correlación de Pearson y la prueba de " $\mathrm{t}$ " para determinar significancia del coeficiente de correlación (Sokal y Rohlf, 1981).

\section{Resultados}

Distribución de tallas. Las tallas analizadas en 1236 ejemplares de $C$. articulatus indicaron una Lt media de 44.1 $\pm 8.5 \mathrm{~mm}$ con un mínimo de $11.3 \mathrm{~mm}$ y un máximo de $86.8 \mathrm{~mm}$. Acerca de la An se tiene un mínimo de $7.1 \mathrm{~mm}$ y un máximo de $78.5 \mathrm{~mm}$, con un promedio de $27.0 \pm 5.8 \mathrm{~mm}$. Mientras que para la $\mathrm{Al}$ se registró una media de 9.8 \pm 2.5 $\mathrm{mm}$ con una talla mínima de $2.0 \mathrm{~mm}$ y una máxima de $29.8 \mathrm{~mm}$. El Pt registrado indicó valores entre 0.1 y 53.8 $\mathrm{g}$ con un promedio de $7.5 \pm 4.7 \mathrm{~g}$ (Cuadro 1). Es evidente una distribución de tallas distinta entre isla Venados e isla Pájaros. La zona protegida de isla Venados registra las tallas más pequeñas, de 11.3 a $63.2 \mathrm{de} \mathrm{mm} \mathrm{Lt}$, con una longitud media de $39.4 \pm 7.3 \mathrm{~mm}$. La zona expuesta de esta isla y la zona protegida de la isla Pájaros presentan tallas intermedias, con intervalos de 13.1 a 68.2 ( $\bar{X}=42.9 \pm 6.9$ ) a 17.2 y 78.9 ( $\bar{X}=42.6 \pm 9.2) \mathrm{mm}$ de $\mathrm{Lt}$, respectivamente. En la zona expuesta de isla Pájaros se registraron tallas de 22.1 a $86.8 \mathrm{~mm}$ de $\mathrm{Lt}$, con una longitud media de $51.7 \pm 10.7$ mm (Fig. 2).

Esta diversidad de tallas registradas se confirma mediante un diagrama de caja y bigotes (Fig. 3). Este diagrama sugiere que existen 3 grupos de tallas entre los quitones colectados; uno en la zona protegida de la isla Venados, otro conjuntamente entre la zona expuesta de esta isla y la zona protegida de la isla Pájaros, y un tercer grupo que corresponde a la zona expuesta de esta última isla.Las pruebas de normalidad de Lilliefors y homocedasticidad de Bartlett indican que solamente los datos Lt de la isla Pájaros en conjunto y la zona protegida de esta misma isla no se distribuyen normalmente $(p<0.01)$ y que los datos registrados presentan varianzas diferentes $(p<0.01)$, debido a que no existe homocedasticidad. En este contexto, el análisis de varianza de una vía de Kruskal-Wallis demostró que existe diferencia significativa entre los promedios de las longitudes de las zonas de colecta $(p<0.01)$. La prueba de comparaciones múltiples no paramétrica de Dunn indicó que no existe diferencia significativa entre los promedios de las longitudes de la zona expuesta de isla Venados y la zona protegida de isla Pájaros (Cuadro 2).

Asimismo, la prueba $U$ de Mann-Whitney estableció que existen diferencias significativas entre las tallas de los quitones colectados en isla Venados e isla Pájaros 
Cuadro 1.Valores de los ejemplares de Chiton articulatus recolectados en la zona protegida (ZP) y expuesta (ZE) de las islas Venados y Pájaros de la bahía de Mazatlán, Sinaloa, México

\begin{tabular}{|c|c|c|c|c|}
\hline \multicolumn{2}{|c|}{ Isla Venados } & \multicolumn{2}{|c|}{ Isla Pájaros } & \multirow[b]{2}{*}{$Z E$} \\
\hline Estadístico & $Z P$ & $\begin{array}{c}Z E \\
\text { Longitud } \\
\text { (mm) }\end{array}$ & $Z P$ & \\
\hline Media & 39.4 & 42.9 & 42.6 & 51.7 \\
\hline Moda & 37.4 & 40.9 & 36.7 & 47.8 \\
\hline Mínimo & 11.3 & 13.1 & 17.2 & 22.1 \\
\hline Máximo & 63.2 & 68.2 & 78.9 & 86.8 \\
\hline Desviación estándar & $\begin{array}{c}7.3 \\
\text { Anch }\end{array}$ & 6.9 & 9.2 & 10.7 \\
\hline Media & 25.4 & 26.2 & 26.0 & 30.5 \\
\hline Moda & 21.1 & 21.1 & 31.1 & 31.1 \\
\hline Mínimo & 8.2 & 7.1 & 11.1 & 12.1 \\
\hline Máximo & 40.0 & 42.5 & 46.8 & 78.5 \\
\hline Desviación estándar & $\begin{array}{l}5.0 \\
\text { Altu }\end{array}$ & 5.4 & 6.1 & 6.6 \\
\hline Media & 8.8 & 9.3 & 9.4 & 11.6 \\
\hline Moda & 11.1 & 11.1 & 11.1 & 11.1 \\
\hline Mínimo & 2.0 & 2.1 & 3.1 & 4.1 \\
\hline Máximo & 14.1 & 17.2 & 18.9 & 29.8 \\
\hline Desviación estándar & $\begin{array}{l}2.0 \\
\text { Peso }\end{array}$ & 2.4 & 2.6 & 3.1 \\
\hline Media & 5.6 & 6.6 & 6.7 & 11.3 \\
\hline Moda & 4.4 & 7.8 & 6.0 & 9.3 \\
\hline Mínimo & 0.1 & 0.2 & 0.4 & 0.8 \\
\hline Máximo & 22.2 & 28.2 & 36.9 & 53.8 \\
\hline Desviación estándar & 2.9 & 3.7 & 5.1 & 7.2 \\
\hline Núm. de organismos & 254 & 245 & 227 & 510 \\
\hline
\end{tabular}

$(p<0.05)$, así como entre la zona protegida y zona expuesta de estas islas $(p<0.05)$.

Relaciones biométricas. Al ajustar los datos observados de talla y peso para establecer las relaciones longitudanchura, longitud-altura, altura-anchura, longitud-peso, anchura-peso y altura-peso de C. articulatus, las ecuaciones obtenidas entre las tallas fueron de tipo lineal y entre talla y peso fueron de tipo potencial (Cuadro 3).

En la mayoría de los casos, los índices de correlación (r) obtenidos fueron bastante satisfactorios $(\mathrm{p}<0.05)$, con valores por encima de 0.92 , excepto para relaciones longitud total-anchura y altura-anchura que registraron 0.87 y 0.84 , respectivamente. Los valores de la pendiente (b) de las relaciones entre la talla y el peso fueron de 2.32 en la relación altura-peso total hasta 2.88 en la relación anchura-peso total.
La relación entre el peso total $(\mathrm{Pt})$ y el peso del pie (Pp) de C.articulatus es lineal $(\mathrm{p}<0.05)$, con un índice de correlación ( $\mathrm{r}=0.93)$ bastante satisfactorio. El pie es la parte comestible de los quitones. La proporción que se aprovecha de cada individuo es aproximadamente el $17 \%$ de su peso total. Los valores máximos registrados para el peso de pie fueron aproximadamente de $8 \mathrm{~g}$ y el valor calculado para el individuo que registró el peso total máximo, con $53.8 \mathrm{~g}$, indica un peso del pie cercano a los $9.0 \mathrm{~g}$.

\section{Discusión}

La información que aquí se presenta sobre talla y peso se incluye entre los primeros datos registrados 


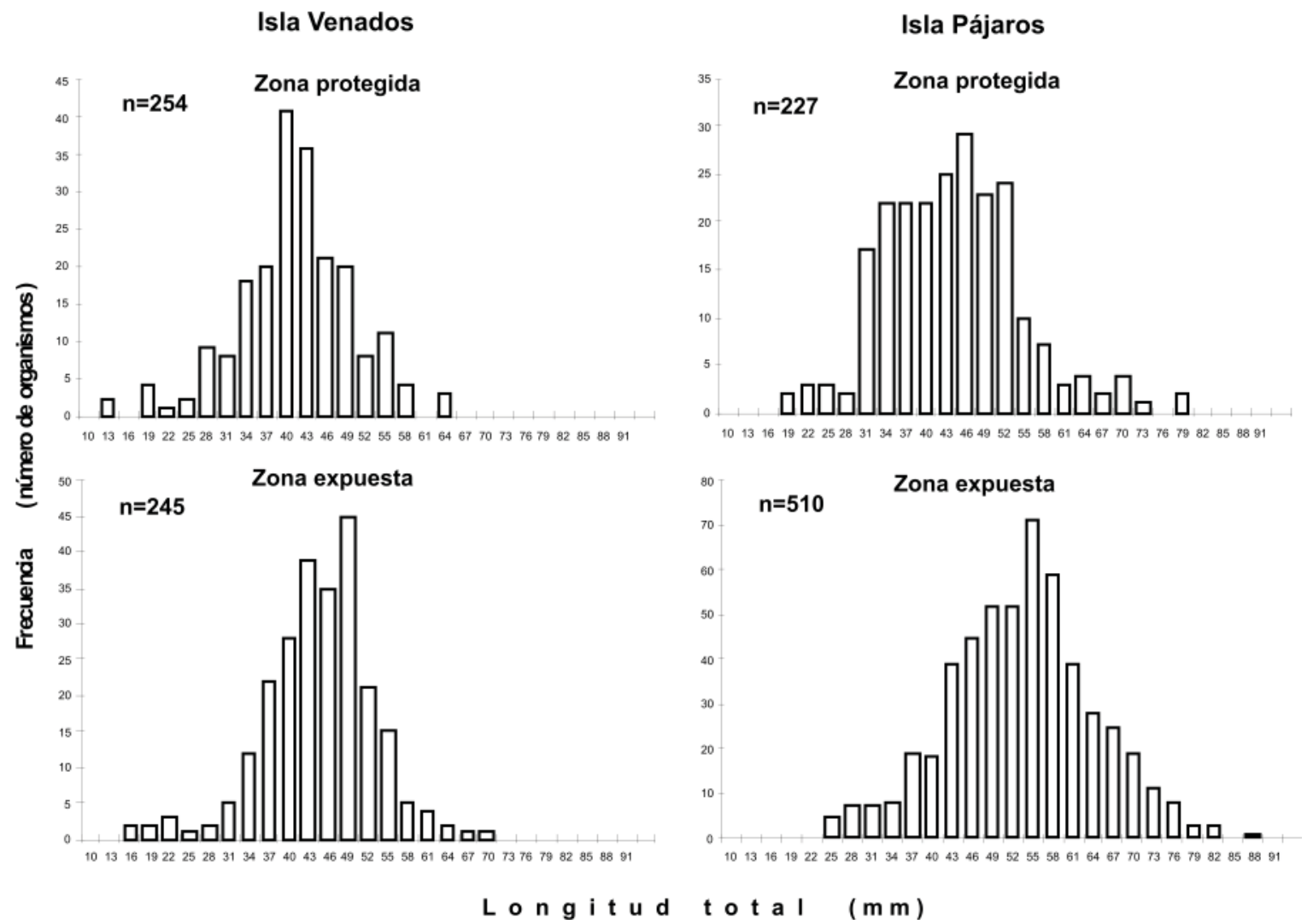

Figura 2. Distribución de tallas de Chiton articulatus en las estaciones de recolecta de las islas Venados y Pájaros de la bahía de Mazatlán, Sinaloa, México.

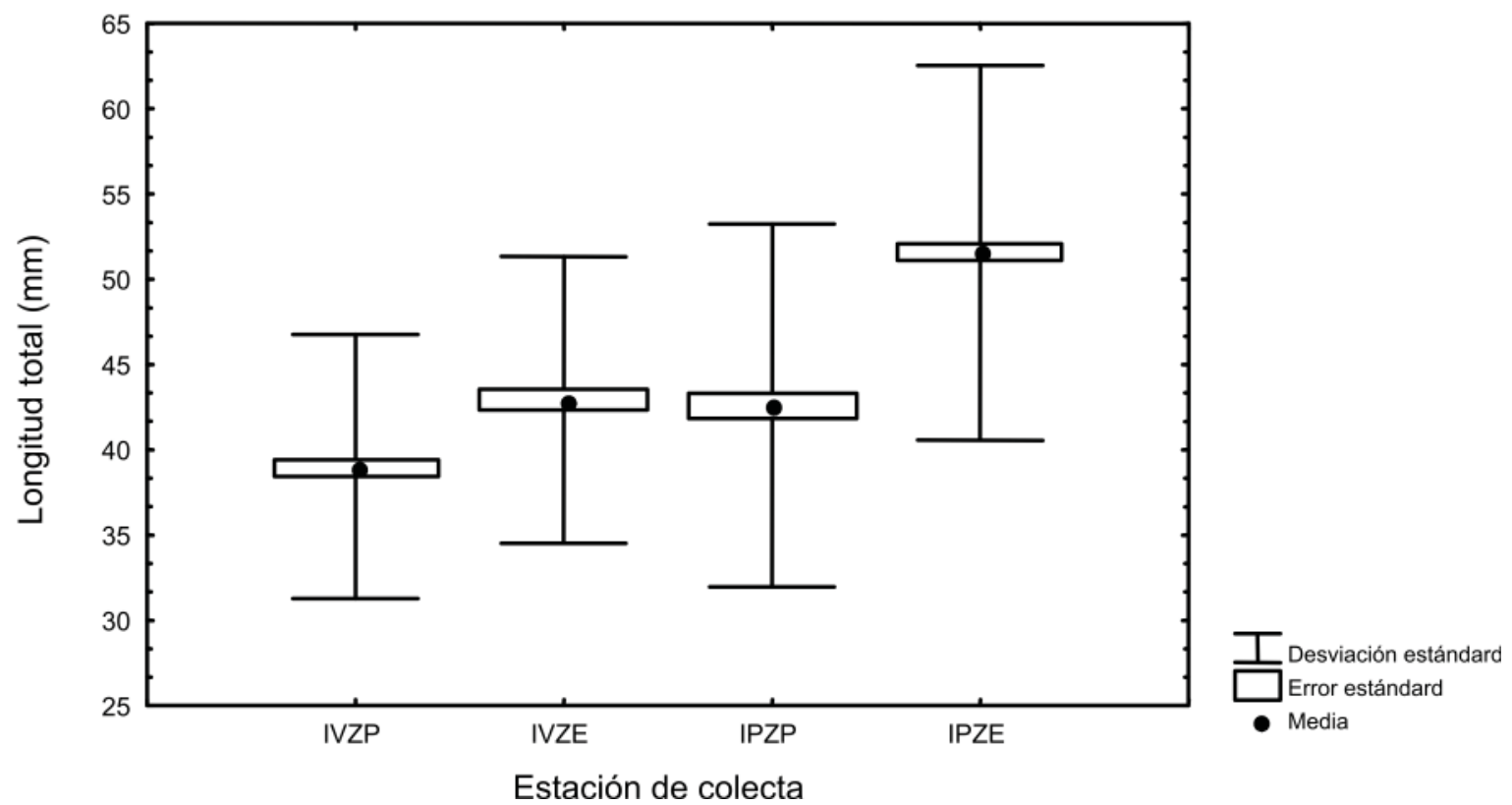

Figura 3. Diagrama de caja y bigote entre los promedios de la longitud total de Chiton articulatus registrados en la zona protegida (ZP) y expuesta (ZE) de las islas Venados (IV) y Pájaros (IP) de la bahía de Mazatlán, Sinaloa, México. 
Cuadro 2. Análisis de comparación múltiple por el método de Dunn aplicado a los datos de longitud total de Chiton articulatus registrados en la zona protegida (ZP) y expuesta (ZE) de las islas Venados (IV) y Pájaros (IP) de la bahía de Mazatlán, Sinaloa, México

\begin{tabular}{ccc}
\hline Estación & $\bar{X}(\mathrm{~mm} \mathrm{de} \mathrm{Lt})$ & $n$ \\
\hline IVZP & $39.4 \pm 7.74^{\mathrm{a}}$ & 254 \\
IVZE & $43.5 \pm 8.4^{\mathrm{b}}$ & 245 \\
IPZP & $42.6 \pm 10.6^{\mathrm{b}}$ & 227 \\
IPZE & $51.7 \pm 10.9^{\mathrm{c}}$ & 510 \\
\hline
\end{tabular}

*Las letras diferentes indican diferencias significativas y las letras iguales indican grupos de datos homogéneos o que no existen diferencias significativas.

en la literatura sobre las dimensiones corporales de $C$. articulatus. De acuerdo con las dimensiones corporales aquí registradas, de 11.3 a $86.8 \mathrm{~mm}$ de longitud total, Chiton articulatus se ubica en un intervalo de tallas común entre los quitones, ya que según Barnes (1977), la mayor parte de las especies tienen de 3 a $12 \mathrm{~cm}$ de longitud, mientras que Keen (1971) menciona que los adultos de esta especie presentan una longitud de 5 a 10 cm. Por su parte, Holguin-Quiñones y Michel-Morfín (2002) registraron tallas de 29.0 a $108.0 \mathrm{~mm}$ de Lt y un peso de 2.0 a $86.5 \mathrm{~g}$ en la isla Socorro del Archipiélago Revillagigedo, México.

Sinembargo, seobservaron diferencias estadísticamente significativas entre los valores promedio y la distribución de tallas de $C$. articulatus entre las distintas estaciones de recolecta de las islas Pájaros y Venados de la bahía de Mazatlán. La talla máxima siempre se obtuvo en la zona expuesta de la isla Pájaros y la talla mínima correspondió a la zona protegida de la isla Venados. En general, se observa una tendencia, en ambas islas, de mayores tallas en la zona expuesta respecto de la protegida.

Al comparar los histogramas de frecuencia de las 4 estaciones de recolecta, además de las diferencias señaladas con anterioridad, también se observa un incremento de las tallas desde la zona protegida hacia la zona expuesta de ambas islas. Las diferencias en talla de especies de moluscos de áreas protegidas y expuestas del intermareal rocoso están documentadas en muchas publicaciones y se explican con base en factores biológicos, físicos y antropogénicos, principalmente en el caso de los gasterópodos (Atkinson y Newbury, 1984; Crothers, 1992; Hobday, 1995; GiraldoLópez y Gómez-Schouben, 1999; Ríos-Jara et al., 2004).

En el presente trabajo, la diferencia entre las tallas puede ser consecuencia de factores externos a la población de C. articulatus, es decir, por la presencia de los turistas y/o visitantes locales, que incluyen a quienes capturan quitones, así como por factores físicos que incluyen el oleaje y la topografía del intermareal rocoso. La zona protegida de la isla Venados con oleaje de baja intensidad y amplias terrazas de abrasión, registra las tallas más pequeñas y es de muy fácil acceso para los turistas y pescadores locales, por lo tanto los quitones ahí presentes son muy vulnerables; según Flores-Campaña et al. (2001), a la playa de la isla Venados llegan en promedio 50 turistas diariamente, y es común que durante sus recorridos por la playa colecten cualquier organismo bentónico (erizos, conchas y quitones, entre otros) de la zona intermareal.

A la zona expuesta de la isla Venados se puede llegar con cierto grado de dificultad, pues se debe recorrer toda su zona protegida hasta llegar a la isla Lobos y luego, a través de El Cuello, pasar hacia su parte expuesta, donde se registraron tallas intermedias, al igual que en la zona protegida de la isla Pájaros, que sólo es accesible en embarcaciones con motor fuera de borda, veleros y kayacks.

Por último, la zona expuesta de la isla Pájaros, que registró las tallas más grandes, es de muy difícil acceso por su accidentada topografía y alto grado de exposición al oleaje, sólo se puede llegar a ella desembarcando en la zona protegida y después hay que atravesar toda la isla, de topografía muy abrupta y empinada, además de abundante vegetación; por lo mismo, la accesibilidad a los quitones de esta zona es muy baja.

Al igual que sobre el registro de las dimensiones corporales, las relaciones biométricas aquí establecidas han sido escasamente descritas con anterioridad para Chiton articulatus, sólo se tiene información sobre la relación longitud total-peso de esta especie en la isla Socorro del archipiélago Revillagigedo, México (Holguín-Quiñones y Michel-Morfín, 2002). En la literatura revisada no se encontró información acerca de las relaciones biométricas de alguna otra de las 600 especies de quitones descritas en los mares del mundo, excepto algunos datos sobre talla y referidos a la longitud total.

Se han realizado análisis de las relaciones biométricas en otras especies de moluscos de la región como en Crassotrea corteziensis, Mytella strigata y Cardita affinis y el caracol de tinte Plicopurpura pansa (Enciso-Enciso et al., 1998). La importancia de estas relaciones biométricas en los quitones, además de la posibilidad de hacer estimaciones para cualquier individuo que por razones de manipulación inadecuada se encuentre incompleto, reside en el hecho de que permite conocer su factor de condición y tipo de crecimiento. Asimismo, las estimaciones de los diferentes coeficientes obtenidos dejan abierta la posibilidad de comparación con los que se obtengan a futuro; aunque las relaciones biométricas descritas en este trabajo deben 
Cuadro 3. Valores de las relaciones biométricas de Chiton articulatus, colectado en las islas Venados y Pájaros de la bahía de Mazatlán, Sinaloa, México

\begin{tabular}{llll}
\hline \multicolumn{1}{c}{ Relación } & $n$ & \multicolumn{1}{c}{ Ecuación } & \multicolumn{1}{c}{$r$} \\
\hline Longitud total-altura & 1,236 & $\mathrm{An}=0.5423(\mathrm{Lt})+2.7594$ & 0.9222 \\
Longitud total-anchura & 1,236 & $\mathrm{Al}=0.2316(\mathrm{Lt})-0.4576$ & 0.8699 \\
Altura-anchura & 1,236 & $\mathrm{Al}=0.3807(\mathrm{An})-0.3832$ & 0.8382 \\
Longitud total-peso total & 1,236 & $\mathrm{Pt}=0.0001(\mathrm{Lt})^{2.8404}$ & 0.9650 \\
Anchura-peso total & 1,236 & $\mathrm{Pt}=0.0005(\mathrm{An})^{2.8807}$ & 0.9334 \\
Altura-peso total & 1,236 & $\mathrm{Pt}=0.0329(\mathrm{Al})^{2.3236}$ & 0.9300 \\
Peso total-peso del pie & 1,236 & $\mathrm{Pt}=4.8778(\mathrm{Pp})+1.0933$ & 0.9352 \\
\hline
\end{tabular}

aplicarse de manera exclusiva para C. articulatus entre las tallas de 11.3 a $86.8 \mathrm{~mm}$ de longitud.

El tipo de crecimiento de los organismos se puede conocer por medio del valor de la pendiente (b), de la ecuación que expresa la relación peso-longitud. Esta constante determina la proporcionalidad de los incrementos de longitud respecto al peso. En especies marinas, el valor de esta constante fluctúa, entre 2 y 4 , un valor igual a 3 indica un crecimiento isométrico; sí es mayor o menor a 3, el crecimiento se considera alométrico (Guzmán, 1987).

Los valores de la pendiente (b) de las relaciones entre la talla y el peso de C. articulatus fueron cercanas a 3, sobre todo en las relaciones peso total-longitud total y peso total-anchura. El comportamiento general observado en el presente trabajo indica que el tipo de crecimiento es isométrico, es decir, los quitones crecen y aumentan de peso proporcionalmente.

Chiton articulatus habita en el substrato rocoso de la franja intermareal, principalmente en la zona de rompientes. Se puede encontrar en grietas, bajo las rocas $\mathrm{y}$ en cualquier otro sustrato duro como conchas. Las tallas que comúnmente alcanza y la relativa abundancia de C. articulatus en las 3 islas de la bahía de Mazatlán, hacen atractiva su captura. La pesca de los quitones en la localidad es meramente artesanal; aunque el lugar donde habitan no es de fácil acceso, si los pescadores se esperan a las mareas más bajas se puede facilitar su captura.

La captura de C. articulatus se realiza manualmente, empleando ganchos, espátulas y cuchillos, ya que estos organismos se encuentran adheridos a las rocas por medio de un pie ancho y plano que ocupa la mayor parte de la superficie ventral. Después de obtener una cantidad importante de quitones, prácticamente en el mismo lugar donde se capturaron, el pie es separado de la concha y del resto del organismo.
Es poca la información que se tiene sobre la pesca de los quitones en el mundo. La captura de C. articulatus se práctica desde hace muchos años en las costas de Mazatlán, Sinaloa, como una actividad de subsistencia que diversos grupos realizan de manera temporal o complementaria a otras actividades. Como no existe ningún tipo de normatividad y/o vigilancia sobre la captura y comercialización de este recurso, es una actividad que libremente puede ejercer cualquier persona.

El tamaño que alcanza C. articulatus hace que sea la especie de quitón que mayormente se captura de manera artesanal por los pescadores locales. Los individuos colectados son utilizados como carnada o comercializados en restaurantes y marisquerías de la localidad para consumo humano. Las conchas de estos quitones también son apreciadas para la fabricación de artesanías, ya que sus placas son muy utilizadas para imitar pétalos de flores.

Algunos restaurantes y marisquerías de la región ofrecen entre sus platillos las cucarachas de mar, como se conoce comúnmente a los quitones, aunque en el menú se indica que sólo se ofrecen en "temporada". El valor comercial de este platillo depende básicamente del tipo de establecimiento. Segúnel valor promediodel pesoregistrado en el presente trabajo, se requieren aproximadamente 588 quitones para completar un kilogramo de este marisco. La indicación de que el platillo sólo se ofrece en temporada, en realidad corresponde a la existencia o no del recurso, lo que se relaciona con las condiciones climatológicas de la región y las necesidades que tengan quienes se dedican a su captura y comercialización.

Se recomienda profundizar en estudios acerca de la biología pesquera de C. articulatus, principalmente de su densidad poblacional, crecimiento y madurez gonádica, así como de los volúmenes de captura, entre otros. Se requiere esta información para establecer los lineamientos 
generales y la normatividad correspondiente, antes de que desaparezcan las escasas poblaciones de este recurso pesquero todavía existentes.

\section{Agradecimientos}

A Manuel Peñuelas Román, Flor Arreola Vergara y Sarai Gamez Torrontegui por su colaboración en la recolecta de los quitones y registros de datos biométricos. A la empresa Aqua Sport Center por las facilidades brindadas durante las visitas de campo a la islas Pájaros y Venados. A Domenico Voltolina Lobina por sus acertadas recomendaciones durante la revisión del manuscrito. Este trabajo fue parcialmente apoyado por el Fondo Mixto Gobierno de Sinaloa-CONACyT (SIN-2006-CO137439).

\section{Literatura citada}

Atkinson, W. D. y S. F. Newbury. 1984. The adaptations of the rouge winkle, Littorina rudis, to desiccation and dislodgement by wind and waves. Journal of Animal Ecology 53:93-106

Barnes, R. D. 1977. Zoología de invertebrados, tercera edición. Nueva Interamericana, México, D.F. 509 p.

Baxter, J. M. y A. M. Jones. 1981. Valve structure and growth in the Chiton lepidochitona cinereus (Polyplacophora:Ischnochitonidae). Biology Science Department, Dundee University 61:65-78.

Brusca, R. C. 1980. Common intertidal invertebrates of the Gulf of California, second edition. The University of Arizona Press. 427 p.

Cameron, R. A. 1982. Effect of gamma-aminobutyric acid on the settlement of larvae of the black chiton Katharina tunicata. Marine Biology 72:243-247.

Cervera-Rodríguez, L., J. H. Álvarez-Hernández y N. E. González. 2004. Riqueza de especies de las clases Poliplacophora y Gastropoda en la laguna arrecifal de Majahual, Quintana Roo, México. In Resúmenes de la IX Reunión Nacional de Malacología y Conquiología, Mérida, Yucatán. L.A. Rodríguez-Gil, (ed.). Instituto Tecnológico de Mérida y Sociedad Mexicana de malacología, A. C. Mérida, Yucatán, p. 125-129.

Clark, R. N. 2000. The chiton fauna of the Gulf of California rhodolith beds (with descriptions of four new species). Neumonia 43:1-8.

Crothers, J. H. 1992. Shell size and shape variation in Littorina littorea (L.) from west Somerset. In Proceedings of the Third International Symposium on Littorinid Biology, J. Grahame, P. J. Mill and D.
G. Reid (eds.). The Malacological Society of London. p. 91-97.

Cruz, R. A. y A. Sotela. 1984. Contribución a la biología de Chiton stockesii (Polyplacophora: Chitonidae) de Punta Pochote, Puntarenas, Costa Rica. Revista de Biología Tropical 32:61-68.

Deshpande, U. D. y R. Nagabhushanam. 1983. Seasonal changes in the biochemical composition of the chiton Chiton iatricus (Polyplacophora: Mollusca) and the marine pulmonate Onchidium verruculatum (Gastropoda: Mollusca) in the relation to their reproductive cycles. Marine Biology 72:227-234.

Enciso-Enciso, C., V. M. Ramírez-Hernández, A. R. Tirado-Nuñez y A. Vallarta-Pérez. 1998. Evaluación de la población del caracol Plicopurpura pansa (Gould, 1853), en Mazatlán, Sinaloa, México. Tesis Facultad de Ciencias del Mar, Universidad Autónoma de Sinaloa, Mazatlán, Sinaloa. 75 p.

Fischer, W., F. Krupp, W. Schneider, C. Sommer, K. Carpenter y V. H. Niem. 1995. Guía FAO para la identificación de especies para los fines de la pesca, Pacífico centro-oriental. I. Plantas e invertebrados, FAO, Roma. 646 p.

Flores-Campaña, L. M., B. Millán-Sánchez, M. A. OrtizArellano y J. F. Arzola-González. 2001. Estructura ecológica, impacto ambiental y aprovechamiento turístico sustentable de las Tres Islas, Mazatlán, Sinaloa. In Turismo sustentable en Sinaloa; lineamientos y estrategias, L.M Flores-Campaña (ed.). UAS, Culiacán, Sinaloa, p. 43-58.

Giraldo-López, A. y C. Gómez-Schouben. 1999. Variación en la concha de Siphonaria gigas (Sowerby, 1825) como respuesta al efecto de la intensidad de las olas. Ciencias Marinas 25:213-224.

González, N. E. 1993. Moluscos endémicos del Pacífico. In Biodiversidad marina y costera de México. SalazarVallejo, S. y N. González (eds.). Comisión Nacional para el Conocimiento y Uso de la Biodiversidad, México, D.F. p. 328-357.

Grayson, J. E. y M. G. Chapman. 2004. Patterns of distribution and abundance of chitons of the genus Ischnochiton in intertidal boulder fields. Austral Ecology 29:363-373.

Guzmán, M. A. 1987. Biología, ecología y pesca del langostino Macrobrachium tenellum (Smith, 1871), en lagunas costeras del estado de Guerrero, México. Tesis doctorado Instituto de Ciencias del Mar y Limnología, Universidad Nacional Autónoma de México. México, D.F. 135 p.

Hendrickx, M. E. y A. Toledano-Granados. 1994. Catálogo de moluscos. Colección de referencia, Estación Mazatlán, ICMyL, UNAM. Comisión Nacional para el 
Conocimiento y Uso de la Biodiversidad, Instituto de Ciencias del Mar y Limnología, Universidad Nacional Autónoma de México, México, D.F. 71 p.

Hobday, A. 1995. Body-size variation exhibited by an intertidal limpet: Influence of wave exposure. Tidal height and migratory behavior. Journal of Experimental Marine Biology and Ecology 189:29-45.

Holguin-Quiñones, O. F. y J. E. Michel-Morfín. 2002. Distribution, density and length-weigth relationship of Chiton articulatus Sowerby, 1832 (MolluscaPolyplacophora) on isla Socorro, Revillagigedo Archipelago, México. Journal of Shellfish Research 21:239-241.

Kaas, P. y R. A. van Belle. 1998. Catalogue of living chitons (Mollusca: Polyplacophora), second edition. Backhuys, Rotterdam. 204 p.

Keen, M.A. 1971. Seashells of tropical west America (marine mollusks from Baja California to Perú). Houghtan Mifflin, Boston, Massachusetts. 1064 p.

Kelaher, B. P. y V. J. Cole. 2005. Variation in abundance and size structure of populations of the small chiton Acanthochiton retrojecta. Journal of Molluscan Studies 71:145-151.

Montoya, M. 1983. Los moluscos marinos de la Isla del Coco, Costa Rica. I. Lista anotada de especies. Bernesia 21:325-353.

Nagabhushanam, R.y U.D.Deshpande. 1982.Reproductive cycle of the chiton Chiton iatricus and environmental control of its gonad growth. Marine Biology 67:9-13.

O’Neill, M. H. B. 1985. A review of the living New Zealand members of Onithochiton Gray, 1947 (Mollusca: Polyplacophora). New Zealand Journal of Zoology 12:141-154.

Otway, N. M., 1994. Population ecology of the low-shore chitons Onithochiton quercinus and Plaxiphora albida. Marine Biology 121:105-166.
Reguero, M. y A. GarcíaCubas. 1989. Moluscos de la plataforma continental de Nayarit: Sistemática y ecología (cuatro campañas oceanográficas). Anales del Instituto de Ciencias del Mar y Limnología, Universidad Nacional Autónoma de México 16:33-58.

Reyes-Gómez, A. y M. A. Salcedo-Vargas. 2002. The recent Mexican Chiton (Mollusca: Polyplacophora) species. The Festivus 34:17-27.

Reyes-Gómez, A. 2004. Chitons in Mexican waters. Bollettino Malacologico 5:69-82.

Ricker, W. E. 1975. Computation and interpretation of biological statistics of fish populations. Bulletin of Fisheries Research Board of Canada 191:382.

Ríos-Jara, E., C. C. Hernández-Cedillo, E. Juárez-Carrillo e I. Enciso-Padilla. 2004. Variations in density, shellsize and growth with shore height and wave exposure of the rocky intertidal snail, Calyptraea spirata (Forbes, 1852), in tropical Mexican Pacific. Journal of Shellfish Research 23:545-552.

Rojas-Herrera., A. 1988. Análisis biológico-pesquero de la cucaracha de mar (Chiton articulatus Sowerby, 1832) de Acapulco, Guerrero. México. In Memorias del IX Congreso Nacional de Zoología, México. Universidad Autónoma de Juárez, Tabasco y Sociedad Mexicana de Zoología, Villahermosa, Tabasco. p. 151-156.

Sakker, E. R. 1984. Sperm morphology, espermatogenesis and spermiogenesis of three species of Chiton (Mollusca, Polyplacophora). Zoomorphology 104:111-121.

Slieker, F. J. A. 2000. Chitons of the world, an illustrated synopsis of recent Polyplacophora. Mostra Mondiale Malacologia, Cupra Marittima. 154 p.

Sokal, R. R. y F. J. Rohlf. 1981. Biometría: Principios y métodos estadísticos en la investigación biológica. Blume, Madrid. 829 p.

Zar, J. H. 1996. Bioestadistical analysis, tercera edición. Prentice-Hall, Englewood Cliffs, New Jersey. 662 p. 